\title{
Tolerance to Cell Wall-active Antibiotics in Coagulase-negative Staphylococci
}

Tolerance to cell wall-active antibiotics is common in Staphylococcus aureus, having been documented in 36 to $63 \%$ of strains recovered primarily from blood cultures (1-3). Occasional strains of coagulase-negative staphylococci (CNS) have been reported as tolerant $(4,5)$. However, no systematic effort has been made to record the frequency of tolerance in a large number of isolates. Considering the emerging importance of CNS as a cause of nosocomial septicemia in adults and neonates, we studied the incidence of tolerance in $40 \mathrm{CNS}$ strains isolated from blood and cerebrospinal fluid cultures.

For each isolate, minimum inhibitory concentrations (MIC) and minimum bactericidal concentrations (MBC) were determined in duplicate by the broth microdilution method, using Mueller-Hinton (MH) broth as the growth medium. All of the isolates were studied during the stationary phase of growth. Two inoculum densities, $1 \times 10^{5}$ and $1 \times 10^{7}$ colony forming units per $\mathrm{ml}(\mathrm{cfu} / \mathrm{ml})$ were tested. The MIC was defined as the lowest concentration of antibiotic which inhibited visible growth of the organism after either 24 or 48 hours of incubation at $35^{\circ} \mathrm{C}$. The MBC was determined by subculturing from microdilution wells to $\mathrm{MH}$ agar. Tolerance was defined as an MBC:MIC ratio of $\geq 32$. Susceptibility of the organisms to the following antimicrobial agents was determined: sodium oxacillin, vancomycin hydrochloride, cephalothin sodium and imipenem.

Isolates of CNS included in the analysis were identified as Staphylococcus epidermidis (21), Staphylococcus hominis (7), Staphylococcus simulans (6), Staphylococcus warneri (2) and Staphylococcus capitis (1). Three isolates were not identified. Six of the isolates were oxacillin-resistant $(\mathrm{MIC} \geq 4 \mathrm{mg} / \mathrm{l})$ at an inoculum density of $1 \times 10^{5} \mathrm{cfu} / \mathrm{ml}$. After 24 hours of incubation, 13 isolates (33\%) displayed

\section{Literature}

1. Hill, M. D., Venglarcik, J. S., Best, G. K.: Oxacillin-tolerant staphylococcal bacteremia in children. J. Pediatr. 96 (1980) 1035-1037.

2. Mayhall, C. G., Apollo, E.: Effect of storage and changes in bacterial growth phase and antibiotic concentrations on antimicrobial tolerance in Staphylococcus aureus. Antimicrob. Agents Chemother. 18 (1980) 784-788.

3. Sabath, L. D., Wheeler, N., Laverdiere, M., Blazevic, D., Wilkin- tolerance to one or more of the antibiotics at either or both of the inoculum densities tested. Seven strains were tolerant to oxacillin, seven to vancomycin, six to cephalothin and eleven to imipenem. Six isolates displayed tolerance only at an inoculum density of $1 \times 10^{7} \mathrm{cfu} / \mathrm{ml}$. No statistically significant differences in frequency of tolerance were detected among the species of CNS tested. The prevalence of tolerance was identical in oxacillin-sensitive and oxacillin-resistant strains. Only two isolates (5\%) remained tolerant to one or more antibiotics after 48 hours of incubation. For the 11 previously tolerant isolates, the $\mathrm{MBC}$ at 48 hours was less than that at 24 hours and the MBC:MIC ratio was no longer $\geq 32$.

We demonstrated that CNS often are tolerant to cell wallactive antibiotics, at a rate similar to that reported for $S$. aureus. Raising the inoculum density 100 -fold almost doubled the number of isolates displaying tolerance to one or more of the antibiotics tested. A second 24 hours of incubation resulted in the disappearance of the 32-fold difference between inhibitory and killing activities in 11 of the 13 tolerant isolates $(85 \%)$ ). Our study population was not appropriate for comparing the clinical courses in patients infected with tolerant or non-tolerant CNS. Nevertheless, tolerance may yet prove to be important in patients with locally impaired phagocytosis, as in prosthetic device or CSF shunt infections, or systemically impaired phagocytosis as in immunocompromised hosts or newborns.

G. Alpert, J. M. Campos

G. Alpert, M.D., Division of Infectious Diseases, The Children's Hospital of Philadelphia, Philadelphia, Pennsylvania, U.S.A.;

Dr. J. M. Campos, Microbiology Laboratory, The Children's Hospital of Philadelphia, Philadelphia, Pennsylvania, U.S.A.

son, B. J.: A new type of penicillin resistance of Staphylococcus aureus. Lancet I (1977) 443-447.

4. Arthur, J. D., Bass, J. W., Keiser, J. F., Harden, L. B., Brown, S. L.: Nafcillin-tolerant Staphylococcus epidermidis endocarditis. JAMA 247 (1982) 487-488.

5. Schaad, U. B., Nelson, J. D., McCracken, G. H.: Pharmacology and efficacy of vancomycin for staphylococcal infections in children. Rev. Infect. Dis. 3 (1981) Suppl. 282-288. 